\title{
Prion peptide-mediated cellular prion protein overexpression and neuronal cell death can be blocked by aspirin treatment
}

\author{
JAE-KYO JEONG, MYUNG-HEE MOON, JAE-WON SEOL, JAE-SUK SEO, YOU-JIN LEE and SANG-YOUEL PARK
}

\author{
College of Veterinary Medicine, Bio-Safety Research Institute, Center for Healthcare Technology \\ Development, Chonbuk National University, Jeonju, Jeonbuk 561-756, Republic of Korea
}

Received November 22, 2010; Accepted January 25, 2011

DOI: $10.3892 / \mathrm{ijmm} .2011 .626$

\begin{abstract}
Prion diseases are infectious neurodegenerative disorders characterized by the conversion of the cellular prion protein $(\mathrm{PrPc})$ to the misfolded isoform (PrPsc). Prion peptide PrP 106-126 [PrP (106-126)] shares many physiological properties with PrPsc; it is neurotoxic in vitro and in vivo. $\operatorname{PrP}$ (106-126) induces neurotoxicity by the overexpression of PrPc and activation of the mitogen-activated protein (ERK1/2). Aspirin, an anti-inflammatory drug, is a known ERK inhibitor and prevents neurodegenerative disorders including prion diseases. The influence of aspirin treatment on prion proteinmediated neurotoxicity and expression of PrPc were the focus of this study. Cell viability and apoptosis were assessed by crystal violet staining and the TUNEL and DNA fragmentation assays. Apoptosis-associated protein expression of PrPc, p-53, p-ERK1/2, p-p38, Bcl-2 and cleaved-caspase-3 was examined by Western blotting and immunocytochemistry. Aspirin treatment inhibited PrP (106-126)-induced neuronal cell death in SH-SY5Y neuroblastoma cells. In addition, the PrP (106-126)-mediated increase of p-p38, p53, cleaved-caspase-3 and decrease of Bcl-2 expressions were blocked by aspirin and the ERK inhibitor, PR98059. Furthermore, we showed that the PrP (106-126)-mediated increase of PrPc and p-ERK1/2 were inhibited by PD98059 and aspirin. Taken together, these results demonstrate that ERK1/2 is a key modulator of the protective effect of aspirin on PrP-106-126-mediated cellular prion protein overexpression and neurotoxicity and also suggest that aspirin may prevent neuron cell damages caused by the prion peptide.
\end{abstract}

\section{Introduction}

Prion diseases or transmissible spongiform encephalopathies (TSE) are a family of infectious neurodegenerative disorders

Correspondence to: Dr Sang-Youel Park, College of Veterinary Medicine, Chonbuk National University, Jeonju, Jeonbuk 561-756, Republic of Korea

E-mail: sypark@chonbuk.ac.kr

Key words: prion peptide (106-126), oxidative stress, neuronal cell death, ERK1/2 phosphorylation, aspirin characterized by CNS spongiosis, vacuolation, neuronal loss, and microglial activation (1). The main abnormality associated with the pathogenesis of prion disease is the formation of scrapie prion proteins (PrPsc), which are proteinase $\mathrm{K}$-resistant misfolded proteins that are modified forms of the normal cellular prion protein $(\mathrm{PrPc})(2,3)$.

The production of PrPsc and the progression of prion diseases are dependent upon the presence of $\operatorname{PrPc}(4,5)$. Several studies on conditional prion protein gene knock-out mice, have shown that depletion of PrPc protein in neurons could prevent the disease $(4,6)$. These findings emphasize the importance of therapeutic strategies targeting the PrPc protein. Some reports have shown that the PrPsc and the prion protein peptide induced neuronal oxidative injury and increased PrPc protein levels. In addition, PrPc knock-out cells prevented prion-mediated oxidative injury $(4,7)$. In addition, recent studies have shown that glimepiride prevented prion-mediated neurotoxicity by reduction of PrPc expression (8). Thus, any treatment that affects the amount of PrPC expression may also affect prion-mediated neurotoxicity.

The prion protein peptide 106-126 [PrP (106-126)] contains the amino acid residues 106-126 of the cellular prion protein and resides near the $\mathrm{N}$-terminus of PrPsc $(9,10)$. It possesses many characteristics of the entire PrPsc protein (11). PrP (106-126) is frequently used to study PrPsc pathogenesis, as well as aggregation of endogenous $\mathrm{PrPc}$ to an amyloidogenic form that shares several characteristics with PrPsc $(12,13)$. Recent studies have linked the increased phosphorylation of the ERK1/2 pathway to the neurotoxic effects of PrPsc and PrP (106-126) (14,15). In a model of prion disease, overstimulation of the ERK1/2 pathway was correlated with oxidative injury of neuronal and nonneuronal cells by reactive oxygen species (ROS) generation $(15,16)$.

Aspirin is a known anti-inflammatory drug with a broad spectrum of pharmacological activity and multiple sites of action (17). Recent findings suggest that aspirin exerts direct neuroprotective effects, including NF- $\kappa$ B inhibition (18-20). Similarly, one study showed that aspirin prevented prion peptide-induced neurotoxicity by inhibition of NF- $\kappa \mathrm{B}$ (18). Substantial evidence has indicated that ERK $1 / 2$ is also one of the targets of aspirin in neurodegenerative disease (21). However, the effect of aspirin-mediated ERK1/2 inhibition on prion peptide-induced neurotoxicity has not been evaluated. 
Thus, the influence of aspirin-mediated ERK1/2 inhibition on the PrP (106-126)-mediated overexpression of PrPc protein and neuronal cell damage were investigated. The results show that aspirin treatment decreases PrP (106-126)-mediated PrPc over-expression and prevents neuronal cell death by inhibition of ERK signaling. The results of the present study support the view that regulation of ERK $1 / 2$ is a viable therapeutic strategy for neurodegenerative diseases including prion disease.

\section{Materials and methods}

Cell culture. Human neuroblastoma cell lines (SH-SY5Y) were obtained from the American Type Culture collection (ATCC, Rockville, MD, USA). The cells were cultured in Minimum Essential Medium (Hyclone Laboratories, Logan, UT, USA) that contained $10 \%$ fetal bovine serum (FBS) (Invitrogen-Gibco, Grand Island, NY, USA) and gentamycin $(0.1 \mathrm{mg} / \mathrm{ml})$ in a humidified incubator maintained at $37^{\circ} \mathrm{C}$ and $5 \% \mathrm{CO}_{2}$.

$\operatorname{PrP}$ (106-126) treatment. Synthetic PrP (106-126) (sequence, Lys-Thr-Asn-Met-Lys-His-Met-Ala-Gly-Ala-Ala-Ala-AlaGly-Ala-Val-Val-Gly-Gly-Leu-Gly) was synthesized from Peptron (Seoul, Korea). The peptides were dissolved in sterile DMSO at a concentration of $12.5 \mathrm{mM}$ and stored at $-80^{\circ} \mathrm{C}$.

Crystal violet staining. Cell viability was evaluated by crystal violet staining, as previously described (22). Briefly, the cells were stained with staining solution $(0.5 \%$ crystal violet in $30 \%$ ethanol and $3 \%$ formaldehyde) for $10 \mathrm{~min}$ at room temperature (RT), and washed four times with water. The stained cells were lysed with $1 \%$ sodium dodecyl sulfate (SDS) solution, and the absorbance was measured at $550 \mathrm{~nm}$. Cell viability was calculated based on the relative dye intensity compared to controls.

Terminal deoxynucleotidyl transferase dUTP nick end labeling (TUNEL) assay. TUNEL analysis was performed to measure the degree of cellular apoptosis using an in situ Apo-BrdU DNA fragmentation assay kit (BioVision, San Francisco, CA, USA) following the manufacturer's instructions.

DNA fragmentation assay. Cell pellets were suspended in $0.5 \mathrm{ml}$ of $50 \mathrm{mM}$ Tris-HCl buffer ( $\mathrm{pH} \mathrm{8.0)}$ containing $10 \%$ (v/v) NP-40, $20 \mathrm{mM}$ EDTA and $0.5 \%$ (w/v) SDS, and digested with $500 \mu \mathrm{g} / \mathrm{ml}$ proteinase $\mathrm{K}$ for $4 \mathrm{~h}$ at $65^{\circ} \mathrm{C}$. After digestion, the DNA was sequentially extracted with phenol/chloroform $(1: 1, \mathrm{v} / \mathrm{v})$, and precipitated with ethanol at $-20^{\circ} \mathrm{C}$ overnight. Purified DNA was electrophoresed on a $1.5 \%$ agarose gel and visualized with ethidium bromide staining and ultraviolet transillumination.

Immunofluorescence. SH-SY5Y cells were cultured on glass coverslips in a 24-well plate. Cells were washed with phosphate-buffered saline (PBS) and fixed with cold acetone for 90 sec. Cells were then washed with PBS again, blocked with 5\% FBS in Tris-buffered saline with Tween (TBST), and incubated with cleaved caspase- 3 monoclonal antibodies $(2 \mu \mathrm{g} / \mathrm{ml})$ for $48 \mathrm{~h}$ at room temperature $\left(20^{\circ} \mathrm{C}\right)$. Unbound antibody was removed by an additional PBS wash, and cells were incubated with labeled anti-rabbit Alexa Fluor 546 (for cleaved caspase-3) IgG antibody ( $4 \mu \mathrm{g} / \mathrm{ml})$ for $2 \mathrm{~h}$ at room temperature. Finally, cells were mounted with DakoCytomation fluorescent medium and visualized via fluorescence microscopy.

Western blot analysis. SH-SY5Y cells were lysed in lysis buffer (25 mM HEPES, pH 7.4; 100 mM NaCl, 1 mM EDTA, $5 \mathrm{mM} \mathrm{MgCl}, 0.1 \mathrm{mM}$ DTT, and protease inhibitor mixture). The proteins were electrophoretically resolved on a $10-15 \%$ SDS gel, and immunoblotting was performed as previously described. Equal amounts of lysate protein were resolved on a 10-15\% SDS-polyacrylamide gel and electrophoretically transferred to a nitrocellulose membrane. Immunoreactivity was detected through sequential incubation with horseradish peroxidase-conjugated secondary antibodies and ECL reagents. The antibodies used for immunoblotting were p53 (Santa Cruz Biotechnology), Bcl-2 (Santa Cruz Biotechnology), phosphorylated-p38 (p-p38) (Cell Signaling Technology) phosphorylated-ERK (p-ERK) (Cell Signaling Technology), PrPc (Millipore) and $\beta$-actin (Santa Cruz Biotechnology).

\section{Results}

$\operatorname{PrP}$ (106-126)-induced apoptosis is inhibited by aspirin treatment in SH-SY5Y neuroblastoma cells. The influence of aspirin on PrP (106-126)-induced apoptosis in SH-SY5Y cells was determined by crystal violet staining. The SH-SY5Y cells were exposed to aspirin alone or in combination with $\operatorname{PrP}$ (106-126). The cells were responsive to $\operatorname{PrP}$ (106-126) treatment, and aspirin had no effect on cell viability. However, aspirin treatment inhibited PrP (106-126)-induced neuronal cell death (Fig. 1B). These results were confirmed by morphological examination of the cell population (Fig. 1A). Consistent with these results, the DNA fragmentation assay and TUNEL assay also showed that aspirin treatment completely inhibited PrP (106-126)-induced apoptosis (Fig. 1C and D).

Next, we examined whether aspirin had an effect on the protein levels of p53, p38, Bcl-2 and cleaved caspase-3. The SH-SY5Y cells were pretreated with $0,2.5,5$ or $10 \mu \mathrm{M}$ aspirin, and then exposed to $\operatorname{PrP}$ (106-126). The prior peptide-treated cells showed increased p53 and p-p38 protein levels and decreased Bcl-2 protein levels. By contrast, aspirin treatment was associated with decreased p53 and p-p38 protein levels and increased Bcl-2 protein levels in neuronal cells exposed to $\operatorname{PrP}$ (106-126) (Fig. 2A), in a dose-dependent manner. Immunofluorescent analysis using an antibody against cleaved caspase-3 detected the cleaved product in SH-SY5Y cells pretreated with aspirin and exposed to $\operatorname{PrP}$ (106-126) (Fig. 2B). Collectively, these results are consistent with the idea that aspirin treatment decreases PrP (106-126)-mediated apoptosis.

Aspirin treatment prevents $\operatorname{PrP}$ (106-126)-induced apoptosis by regulation of the ERK1/2 signaling pathway in $\mathrm{SH}-\mathrm{SY} 5 \mathrm{Y}$ cells. The phosphorylation of ERK1/2 has been shown to be responsible for the neurotoxicity associated with prion infections (14,15). Aspirin-induced PrP (106-126) resistance was studied by assessing the ERK1/2 activity after PrP (106-126) treatment. As shown in Fig. 3, p-ERK1/2 protein levels were increased after PrP (106-126) treatment, whereas they were inhibited by aspirin or PD98059 (ERK1/2 inhibitor) treatment. 
A.

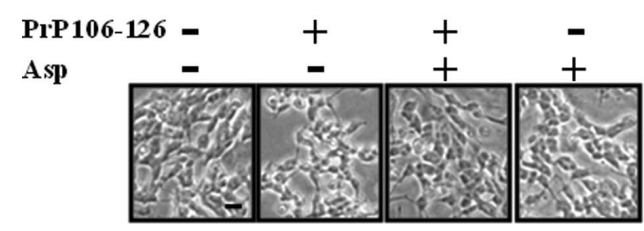

B.

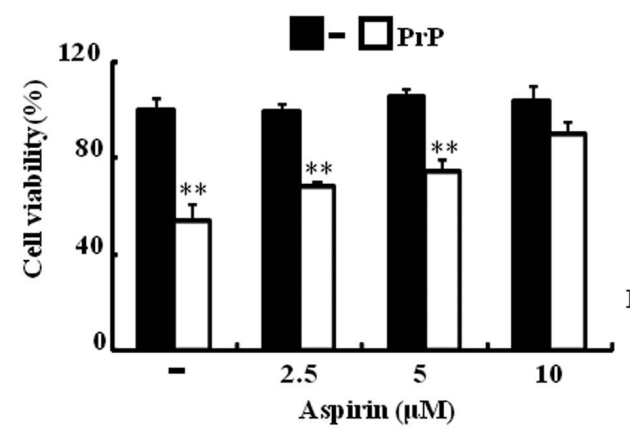

C. PrP106-126 - ++Asp

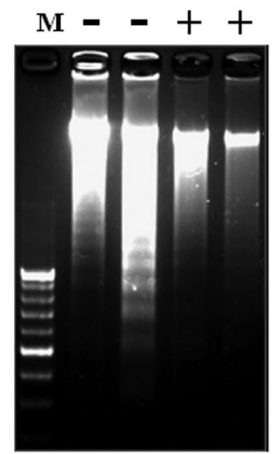

D.

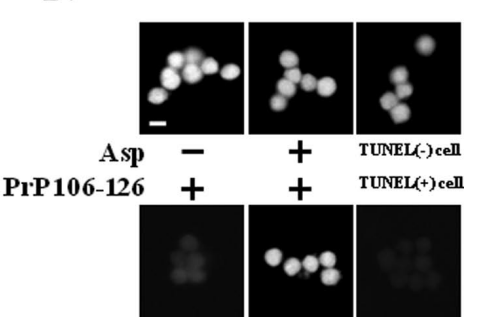

Figure 1. Aspirin protects PrP (106-126)-induced cell death in SH-SY5Y. (A) SH-SY5Y cells were pretreated with 0, 2.5, 5, or 10 $\mu \mathrm{M}$ aspirin for 6 h, and then exposed to $200 \mu \mathrm{M} \operatorname{PrP}$ (106-126) for $42 \mathrm{~h}$. The treated cells were photographed by light microscopy (x200). Scale bar, 100 $\mu \mathrm{m}$ (B) Cell viability was measured by crystal violet staining. Viability of the control cells was set at $100 \%$, and viability relative to the control was measured. The bar graph denotes the mean \pm SEM. $(n=3){ }^{* * *} \mathrm{P}<0.05$. (C) Analysis of genomic DNA isolated from SH-SY5Y cells that were pretreated with $10 \mu \mathrm{M}$ aspirin for $6 \mathrm{~h}$ and then treated with $200 \mu \mathrm{M}$ PrP (106-126) for $42 \mathrm{~h}$. Marker, 100 bp DNA ladder. (D) Representative immunofluorescence images of TUNEL-positive SH-SY5Y cells at $42 \mathrm{~h}$ after exposure to $200 \mu \mathrm{M}$ of $\operatorname{PrP}(106-126)$ in the absence or presence of $10 \mu \mathrm{M}$ aspirin. The cells were counterstained with propidium iodide to show all cell nuclei. Magnification x400; scale bar, $10 \mu \mathrm{m}$.

Since the neurotoxicity of PrPsc and PrP (106-126) has been linked to the overexpression of the PrPc protein (7), we investigated whether aspirin affects the levels of PrPc proteins. The SH-SY5Y cells were pretreated with various concentrations of aspirin, and then exposed to $\operatorname{PrP}$ (106-126). The PrPc protein levels were increased with PrP (106-126) treatment, whereas they were inhibited by aspirin treatment (Fig. 3A). Consistent with these results, the PrP (106-126)induced overexpression of PrPc protein levels was inhibited by PD98059 treatment (Fig. 3B).

To determine whether aspirin had a neuroprotective effect associated with ERK1/2 inactivation in PrP (106-126)-induced neuronal cell death, SH-SY5Y cells were pretreated with aspirin or PD98059, and then exposed to PrP (106-126). The cells were responsive to $\operatorname{PrP}(106-126)$ treatment, while aspirin and PD98059 had no effect on cell viability. However, aspirin and PD98059 treatment inhibited PrP (106-126)-induced neuronal cell death (Fig. 4A). These results were confirmed by morphological examination of the cell population (Fig. 4B). Consistent with these findings, the DNA fragmentation assay and the TUNEL assay showed that aspirin or PD98059 treatment completely inhibited PrP (106-126)-induced apoptosis (Fig. 4C and 4D). Collectively, these results are consistent with the notion that the aspirin-mediated protection from PrP (106-126)-induced apoptosis occurs via regulation of ERK1/2-dependent PrPc expression.

\section{Discussion}

The purpose of this study was to investigate the influence of aspirin treatment on the PrP (106-126)-induced PrPc overstimulation and neuronal cell death. The results of this study suggest the potential of aspirin for therapeutic intervention strategies in neurodegenerative diseases, including prion disease. The PrP (106-126) peptide served as a suitable model for PrPsc neurotoxicity; this is because it possesses many properties of the PrPsc protein $(9-11,16,23)$. PrPsc protein and PrP (106-126) have been shown to induce neuronal cell death by increasing the production of prostaglandin E2 (PGE2) $(8,24,25)$. Some studies have reported that PGE2 production is regulated by p53, p-p38, and ERK1/2 activation $(26,27)$. Consistent with this, PrP (106-126) treatment of SH-SY5Y cells increased p53, p-p38, cleaved caspase-3 and p-ERK protein levels and decreased Bcl-2 protein levels (Figs. 2 and 3A).

A few studies have demonstrated that aspirin treatment influences the progression of prion disease $(18,28)$. Bate et al observed that non-steroidal anti-inflammatory drugs (NSAIDs) protected against prion-induced neurotoxicity by inhibition of PGE2 production (28). Consistent with this, $\mathrm{PrP}$ (106-126)-mediated elevation of p53, p-p38, cleaved caspase-3 and p-ERK protein levels were decreased by aspirin treatment (Figs. 2 and 3A). Taken together, this data suggest that aspirin prevented prion peptide-induced neuronal cell death by the inhibition of p53, p-p38 and p-ERK protein levels.

One report has shown that PrPc overstimulation is a key factor of PrPsc or prion peptide-mediated neurotoxicity (7). Pietri et al suggested that PrP (106-126)-induced neurotoxicity is caused by overstimulation of PrPc-associated signaling pathways, including oxidative stress conditions (7). Using Western blot analysis, we found that aspirin inhibited PrP (106-126)-mediated over-stimulation of PrPc protein levels (Fig. 3A) and blocked neuronal cell death (Fig. 1A). 

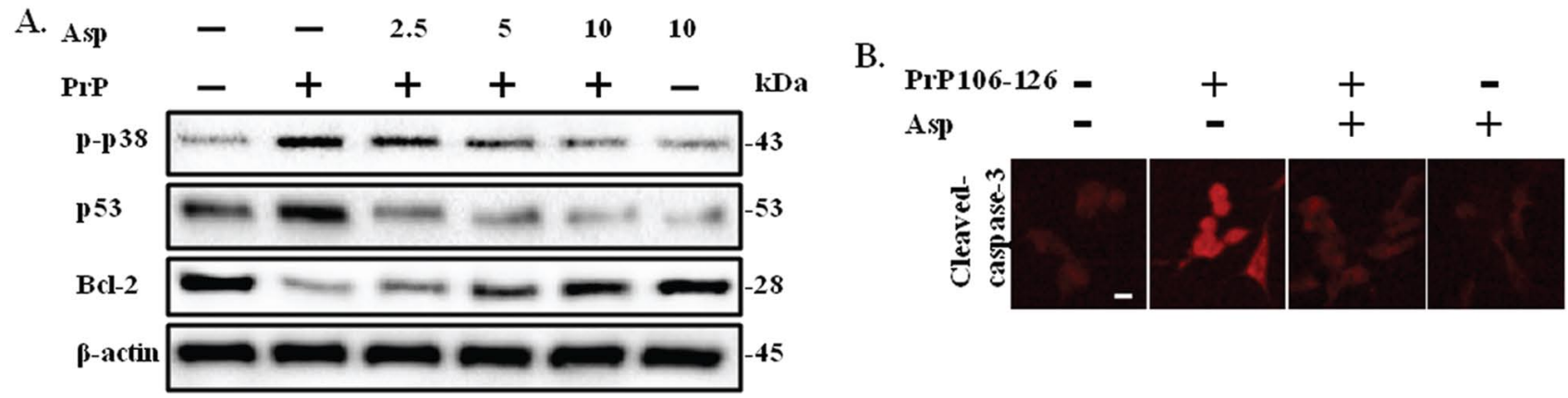

Figure 2. Aspirin regulates the expression levels of apoptosis-related proteins in PrP (106-126)-treated SH-SY5Y cells. (A) SH-SY5Y cells were pretreated with $0,2.5,5$ or $10 \mu \mathrm{M}$ aspirin for $6 \mathrm{~h}$, and then exposed to $200 \mu \mathrm{M} \operatorname{PrP}(106-126)$ for $24 \mathrm{~h}$. The treated cells were assessed for p-p38, p53 and Bcl-2 protein levels by Western blot analysis. Results were normalized to $\beta$-actin. (B) SH-SY5Y cells were pretreated with aspirin (10 $\mu \mathrm{M})$ for $6 \mathrm{~h}$, and then exposed to $200 \mu \mathrm{M} \operatorname{PrP}(106-126)$ for $24 \mathrm{~h}$. The treated cells were immunostained with cleaved caspase-3 (red), as described in Materials and methods, and observed with a fluorescence microscope. Magnification x400; scale bar, $10 \mu \mathrm{m}$.
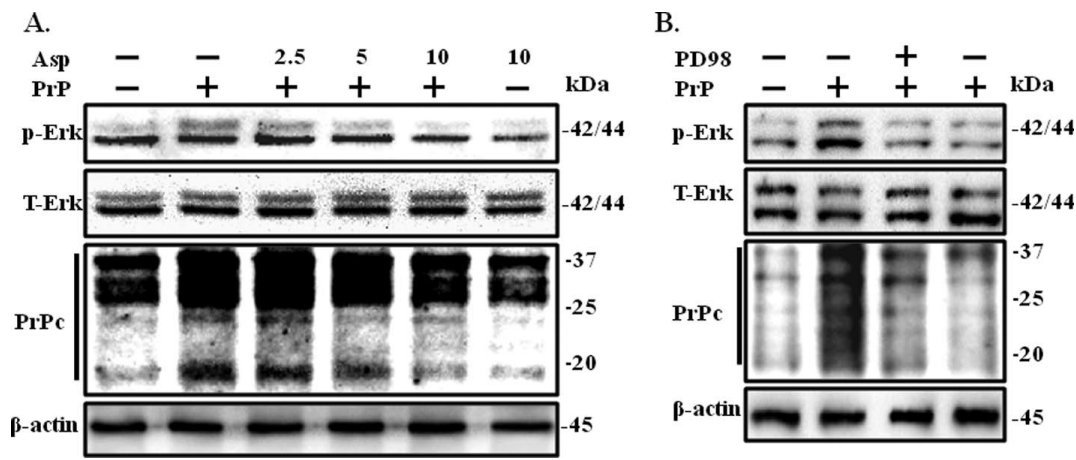

Figure 3. Aspirin inhibits the protein expression of PrPc protein by inhibition of ERK phosphorylation. (A) SH-SY5Y cells were pretreated with a range of aspirin concentrations $(0,2.5,5$ or $10 \mu \mathrm{M})$ for $6 \mathrm{~h}$, and then exposed to $200 \mu \mathrm{M} \operatorname{PrP}(106-126)$ for $24 \mathrm{~h}$. The treated cells were assessed for p-ERK, total-ERK (T-ERK) and PrPc levels by Western blot analysis. Results were normalized to $\beta$-actin (B) SH-SY5Y cells were pre-treated with $10 \mu \mathrm{M}$ aspirin $(6 \mathrm{~h})$ or $10 \mu \mathrm{M}$ PD98059, and then exposed to $200 \mu \mathrm{M} \operatorname{PrP}(106-126)$ for $24 \mathrm{~h}$. Western blot analysis of p-ERK, T-ERK and PrPc levels in SH-SY5Y cells. Results were normalized to $\beta$-actin.

A.

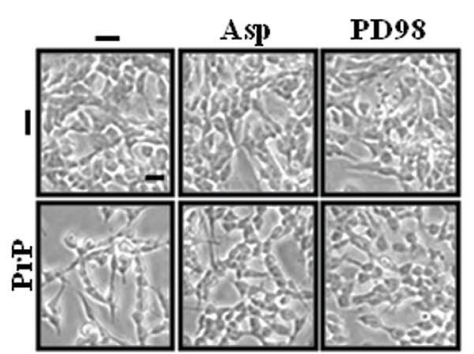

B.

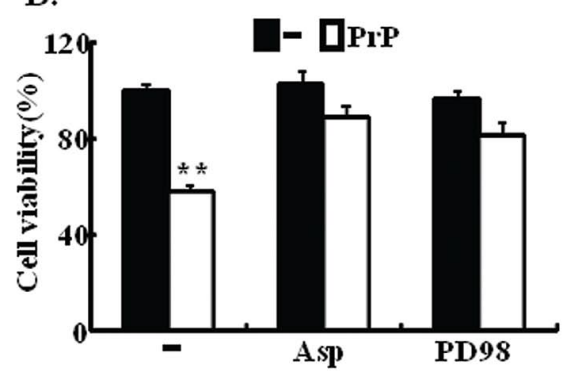

C.

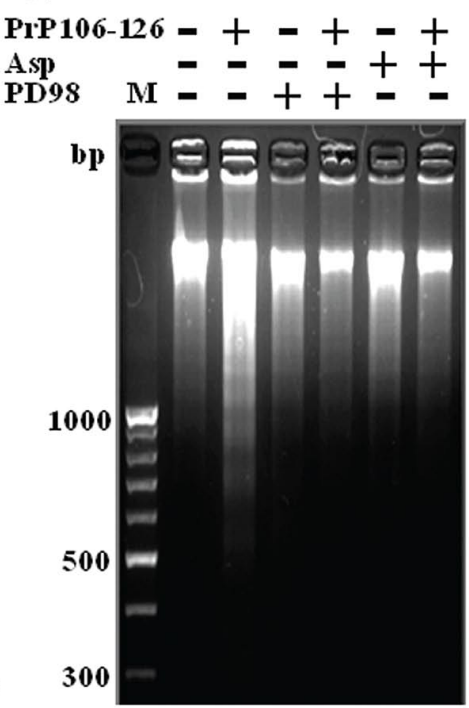

D.

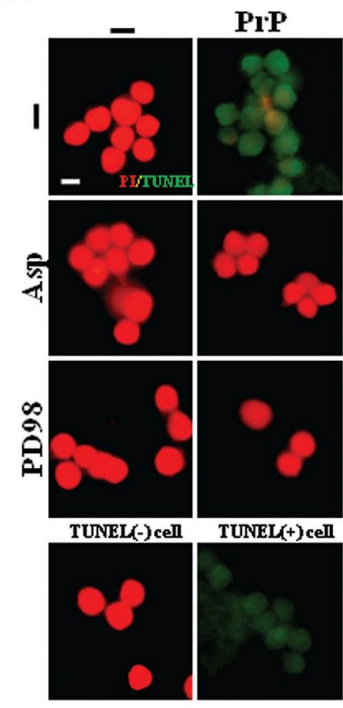

Figure 4. Aspirin protects PrP (106-126)-induced neuronal cell death by inhibition of ERK activation. (A) SH-SY5Y cells were pretreated with aspirin $(10 \mu \mathrm{M})$ or PD98059 $(10 \mu \mathrm{M})$ for $6 \mathrm{~h}$ before $\operatorname{PrP}(106-126)(200 \mu \mathrm{M})$ treatment for $36 \mathrm{~h}$. The treated cells were photographed by light microscopy (x200). Scale bar, $100 \mu \mathrm{m}$. (B) Cell viability was measured by crystal violet staining. Viability of control cells was set at 100\%, and viability relative to the control was measured. The bar graph denotes the mean \pm SEM $(n=3) .{ }^{* *} \mathrm{P}<0.05$. (C) Analysis of genomic DNA isolated from SH-SY5Y cells treated as described in (A). Marker, 100 bp DNA ladder. (D) Representative immunofluorescence images of TUNEL-positive (green) SH-SY5Y cells at $42 \mathrm{~h}$ after exposure to $200 \mu \mathrm{M}$ of PrP (106-126) in the absence or presence of $10 \mu \mathrm{M}$ aspirin $(6 \mathrm{~h})$ or $10 \mu \mathrm{M}$ PD98059 (6 h). The cells were counterstained with propidium iodide (red) to show all cell nuclei. Magnification x400; scale bar, $10 \mu \mathrm{m}$. 
Aspirin is a known anti-inflammatory drug and ERK inhibitor $(17,21)$. Thus, we investigated the effect of aspirinmediated ERK1/2 inhibition on prion peptide-induced PrPc expression. The results of this study showed that $\operatorname{PrP}(106-126)-$ induced PrPc overexpression was inhibited by treatment with aspirin and the ERK inhibitor (PD98059) (Fig. 3A, C and D). In addition, treatment of PD98059 or aspirin protected the cells from PrP (106-126)-induced neurotoxicity (Fig. 4).

Thus, the observations noted in this study support the notion that aspirin treatment protects cells from prion peptidemediated apoptosis by regulation of ERK1/2-mediated PrPc expression. In addition, these results suggest that ERK1/2 inhibitors may be clinically useful for the treatment of neurodegenerative diseases, including prion disease.

\section{Acknowledgements}

This study was supported by a National Research Foundation of Korea grant funded by the Korean Government (20090076873) and the Regional Research Universities Program and the Center for Healthcare Technology Development.

\section{References}

1. Kovacs GG and Budka H: Prion diseases: from protein to cell pathology. Am J Pathol 172: 555-565, 2008.

2. Laurent M and Johannin G: Molecular clues to pathogenesis in prion diseases. Histol Histopathol 12: 583-594, 1997.

3. Kretzschmar HA: Molecular pathogenesis of prion diseases. Eur Arch Psychiatry Clin Neurosci 249 (Suppl. 3): 56-63, 1999.

4. Mallucci G, Dickinson A, Linehan J, Klohn PC, Brandner S and Collinge J: Depleting neuronal $\mathrm{PrP}$ in prion infection prevents disease and reverses spongiosis. Science 302: 871-874, 2003.

5. Manson JC, Clarke AR, McBride PA, McConnell I and Hope J: PrP gene dosage determines the timing but not the final intensity or distribution of lesions in scrapie pathology. Neurodegeneration 3: 331-340, 1994.

6. Bueler H, Aguzzi A, Sailer A, et al: Mice devoid of PrP are resistant to scrapie. Cell 73: 1339-1347, 1993

7. Pietri M, Caprini A, Mouillet-Richard S, et al: Overstimulation of PrPC signaling pathways by prion peptide 106-126 causes oxidative injury of bioaminergic neuronal cells. J Biol Chem 281: 28470-28479, 2006.

8. Bate C, Tayebi M, Diomede L, Salmona M and Williams A: Glimepiride reduces the expression of PrPc, prevents PrPSc formation and protects against prion mediated neurotoxicity in cell lines. PLoS One 4: e8221, 2009.

9. Gu Y, Fujioka H, Mishra RS, Li R and Singh N: Prion peptide 106-126 modulates the aggregation of cellular prion protein and induces the synthesis of potentially neurotoxic transmembrane PrP. J Biol Chem 277: 2275-2286, 2002.

10. La Mendola D, Pietropaolo A, Pappalardo G, Zannoni C and Rizzarelli E: Prion proteins leading to neurodegeneration. Curr Alzheimer Res 5: 579-590, 2008.
11. Singh N, Gu Y, Bose S, Kalepu S, Mishra RS and Verghese S: Prion peptide 106-126 as a model for prion replication and neurotoxicity. Front Biosci 7: a60-a71, 2002.

12. Brown DR: PrPSc-like prion protein peptide inhibits the function of cellular prion protein. Biochem J 352: 511-518, 2000.

13. Forloni G, Bugiani O, Tagliavini F and Salmona M: Apoptosismediated neurotoxicity induced by beta-amyloid and $\operatorname{PrP}$ fragments. Mol Chem Neuropathol 28: 163-171, 1996.

14. Didonna A and Legname G: Aberrant ERK 1/2 complex activation and localization in scrapie-infected GT1-1 cells. Mol Neurodegener 5: 29, 2010.

15. Schneider B, Mutel V, Pietri M, Ermonval M, Mouillet-Richard S and Kellermann O: NADPH oxidase and extracellular regulated kinases $1 / 2$ are targets of prion protein signaling in neuronal and nonneuronal cells. Proc Natl Acad Sci USA 100: 13326-13331, 2003.

16. Jeong JK, Seol JW, Moon MH, et al: Cellular cholesterol enrichment prevents prion peptide-induced neuron cell damages. Biochem Biophys Res Commun 401: 516-520, 2010.

17. Vane JR and Botting RM: Anti-inflammatory drugs and their mechanism of action. Inflamm Res 47 (Suppl. 2): S78-S87, 1998.

18. Yang L, Zhou X, Yang J, Yin X, Han L and Zhao D: Aspirin inhibits cytotoxicity of prion peptide PrP106-126 to neuronal cells associated with microglia activation in vitro. J Neuroimmunol 199: 10-17, 2008.

19. Blanco A, Alvarez S, Fresno M and Munoz-Fernandez MA: Amyloid- $\beta$ induces cyclooxygenase- 2 and PGE2 release in human astrocytes in NF- $\kappa \mathrm{B}$ dependent manner. J Alzheimers Dis 22: 493-505, 2010.

20. Gao F, Bales KR, Dodel RC, et al: NF-kappaB mediates IL-1beta-induced synthesis/release of alpha2-macroglobulin in a human glial cell line. Brain Res Mol Brain Res 105: 108-114, 2002.

21. Pan MR, Chang HC and Hung WC: Non-steroidal antiinflammatory drugs suppress the ERK signaling pathway via block of Ras/c-Raf interaction and activation of MAP kinase phosphatases. Cell Signal 20: 1134-1141, 2008.

22. Seo JS, Seol JW, Moon MH, Jeong JK, Lee YJ and Park SY: Hypoxia protects neuronal cells from human prion protein fragment-induced apoptosis. J Neurochem 112: 715-722, 2010.

23. Forloni G, Angeretti N, Chiesa R, et al: Neurotoxicity of a prion protein fragment. Nature 362: 543-546, 1993.

24. Minghetti L and Pocchiari M: Cyclooxygenase-2, prostaglandin E2, and microglial activation in prion diseases. Int Rev Neurobiol 82: 265-275, 2007.

25. Bate C, Tayebi M, Diomede L, Salmona M and Williams A: Docosahexaenoic and eicosapentaenoic acids increase prion formation in neuronal cells. BMC Biol 6: 39, 2008.

26. Eliopoulos AG, Dumitru CD, Wang CC, Cho J and Tsichlis PN: Induction of COX-2 by LPS in macrophages is regulated by Tpl2dependent CREB activation signals. EMBO J 21: 4831-4840, 2002.

27. Faour WH, He Q, Mancini A, Jovanovic D, Antoniou J and Di Battista JA: Prostaglandin E2 stimulates p53 transactivational activity through specific serine 15 phosphorylation in human synovial fibroblasts. Role in suppression of c/EBP/ NF- $\kappa$ B-mediated MEKK1-induced MMP-1 expression. J Biol Chem 281: 19849-19860, 2006.

28. Bate C, Rutherford S, Gravenor M, Reid S and Williams A: Cyclo-oxygenase inhibitors protect against prion-induced neurotoxicity in vitro. Neuroreport 13: 1933-1938, 2002. 